\title{
Development of a Residential Space-Heating Demand Prediction Method under Uncertainly
}

\author{
Dianzheng $\mathrm{Fu}^{1,2, *}$, Zeyu Zheng ${ }^{1,2}$, Yiming Tong ${ }^{1,2}$ and $\mathrm{Yang} \mathrm{Fu}^{1,2}$ \\ ${ }^{1}$ Shenyang Institute of Automation, Chinese Academy of Sciences, Shenyang, 110016, PR China \\ ${ }^{2}$ Key Laboratory of Network Control System, Chinese Academy of Sciences, Shenyang, 110016, PR China \\ *Corresponding author
}

\begin{abstract}
-in this paper, a prediction method was advanced for residential space heating demand under the fuzz environment. This developed method improved the traditional heat load duration curve method via the quantification expression technique for the fuzzy heating durations. The results indicate that the higher the system possibility is, the narrower the interval of the heat demand prediction result becomes, in which the decision maker can adjust the practical heat provisions of the heat sources. Besides, the opposite heat provision variation trend of the main heat source and peak-shaving heat source can be obtained with the rising thermalization coefficient. Compared with the traditional heat load duration curve method, it is helpful for the relative multi-heat-source centralized heating system managers by providing a potential tradeoff analysis between the system possibility and the selectable heat provision ranges of different heat sources.
\end{abstract}

Keywords-heat demand prediction; uncertainty; heating system; fuzzy set

\section{INTRODUCTION}

The heat supply quality plays a significant role in the life satisfaction degree for the dwellers lived in middle- and higherlatitude areas during the heating period. Nowadays, the urgent expectation for acquiring stringent GHG (i.e. greenhouse gas) and AP (i.e. atmospheric pollutant) emission targets with concurrent high-effective energy utilization prompts the space heating demand predictions of the dwellers to be extremely important [1]. That's mainly for the reason that the relatively conservative heat demand prediction may satisfy the dwellers' desire for the comfortable life during heating season, while potentially cause relatively high GHG and AP emissions and heating cost. Conversely, the positive prediction can alleviate the environmental pollution (i.e. lower the GHG and AP emissions) and heating cost, along with the concurrent declining residential satisfaction degree of heating. Therefore, in recent years, a number of researches have been conducted for the space heating demand predictions by different state-ofthe-art techniques and means in order to provide the reasonable prediction results. For instance, Milan et al. designed the model for heat load prediction, based on the SVR (support vector regression) with a polynomial (SVR-POLY), which is outperformed the results of the SVR with a radial basis function (SVR-RBF) as the kernel function [2]. At the same time, Shamshirband et al. employed adaptive neuro-fuzzy inferences system (ANFIS) to predict the heat load for individual consumers in district heating systems, and presented well-done performance [3]. In comparison with artificial neural networks and genetic programming, Izadyar et al. provided the relatively accurate technique named extreme learning machine (ELM) for forecasting residential heating demand of a case study (Baharestan town, Karaj) in Iran [4]. Catalina et al. applied a multiple regression model, based on the main factors that influence a building's heat consumption, to predict the heating energy demand [5]. A detailed error analysis showed that the model presents a very good accuracy. Likewise, using descriptive statistics and multiple regression methods, Majcen et al. proposed improvements to the current theoretical heating energy consumption calculation for the existing dwellings in Netherlands [6]. It can be concluded from the above studies that most of them employ the relatively complicated model, such as machine learning, physical and statistic modeling, which are totally based on the mass deterministic data inputs. However, such data-support methods may create the cognitive barriers, especially for the heating area lack of history meteorological and building data, to predict the space heat demand during the heating period. Moreover, the uncertainties embedded within the heating duration caused by the climate change further limit the application of the complicated models. Thus, the aim of this research is at advancing a heat demand prediction method under uncertainty, which meanwhile requires a relatively less amount of data. Furthermore, the developed method can not only be utilized in single-heatsource district heating system (SHSCHS), but can also be applied in multi-heat-source centralized heating system (MHSCHS) with directly generating respective residential heat demand parts undertaken by the different heat sources.

\section{Methodology}

\section{A. Heat Load Duration Curve Method}

The heat load duration curve (HLDC) method proposed by $\mathrm{Xu}$ and $\mathrm{He}$ offers a relatively simple but effective method to the heating areas without detailed meteorological and building data for achieving the space heat demands [7]. The detailed calculation formulas can be expressed as follows:

(1) Temperature function:

$$
t_{w}=\left\{\begin{array}{lc}
t_{w}^{\prime} & N \leq 5 \\
t_{w}^{\prime}+\left(5-t_{w}^{\prime}\right) R_{n}^{b} & 5 \leq N \leq N_{z h}
\end{array}\right.
$$


(2) Space-heating load function:

$$
Q_{n}= \begin{cases}Q_{n}^{\prime} & N \leq 5 \\ \left(1-\beta_{0} R_{n}^{b}\right) Q_{n}^{\prime} & 5 \leq N \leq N_{z h}\end{cases}
$$

(3) Relative parameters:

$$
\begin{gathered}
\bar{Q}=\frac{Q_{n}}{Q_{n}^{\prime}}=\frac{t_{n}-t_{w}}{t_{n}-t_{w}^{\prime}} \\
R_{n}=\frac{N-5}{N_{z h}-5} \\
b=\frac{5-\mu t_{p \cdot j}}{\mu t_{p \cdot j}-t_{w}^{\prime}} \\
\mu=\frac{N_{z h}}{N_{z h}-5} \\
\beta_{0}=\frac{5-t_{w}^{\prime}}{t_{n}-t_{w}^{\prime}}
\end{gathered}
$$

(4) Total heat demand undertaken by the MHSCHS can be calculated as follows:

$$
Q_{n n}=24 Q_{n}^{\prime}\left[N_{z h}-\frac{\beta_{0}\left(N_{z h}-5\right)}{1+b}\right]
$$

where $\bar{Q}$ stands for relative space heating load, p.u.; $Q_{n}^{\prime}$ represents total design space heating load, MW; $t_{w}^{\prime}$ represents design outdoor temperature, ${ }^{\circ} \mathrm{C} ; Q_{n}$ means design space heating load corresponding to outdoor temperature, $\mathrm{MW} ; t_{w}$ is outdoor temperature, ${ }^{\circ} \mathrm{C} ; t_{n}$ is design indoor temperature, ${ }^{\circ} \mathrm{C} ; t_{p . j}$ is outdoor average temperature of the space-heating season, ${ }^{\circ} \mathrm{C} ; \mathrm{N}$ means cumulative heating duration corresponding to outdoor temperature, $\mathrm{d} ; N_{z h}$ is the total heating duration, $\mathrm{d} ; b$ stands for exponential value of $R_{n}$, p.u.; $\beta_{0}$ means temperature correction coefficient, p.u.; $\mu$ represents heating duration correction coefficient, p.u.; $R_{n}$ is non-dimensional heating duration, p.u.; Italic subscripts $i$ and $j$ are the heating sub-region and energy conservation type of the building, respectively; $Q_{n n}$ denotes total heat demand undertaken by the MHSCHS, PJ.

\section{B. Heat Demand Prediction under Uncertainty}

Through the above method, the heat demand parts undertaken by different heat sources can be computed by integrating heat load duration curve over the heating duration (i.e. computing the corresponding polygon area under different thermalization coefficients). However, the parameter $N_{z h}$ (i.e. total heating duration) may fluctuate within a range, depending on the annual practical meteorological condition, and the heat demand bias could exist if $N_{z h}$ is annually considered as a fixed level. Therefore, when the annual heating project is constructed for dwellers, the parameter $N_{z h}$ evaluation should be conducted by the experts and stakeholders according to annual meteorological characteristics, causing the fuzzy uncertainty penetration into $N_{z h}$ consequently. The fuzzy heat duration can be expressed as isosceles trapezoid, irregular trapezoid or triangular fuzzy numbers, depending on the practical meteorology evaluation of the year. In order to steer clear of the complicated fuzzy algorithm, a feasible fuzzy heat demand prediction method is provided according to a significant linearity approximation in (2) [8]. The detailed formulation process can be elucidated in Figure 1 with the SHSCHS as the example. The corresponding steps include:

Step 1: Provide the fuzzy $N_{z h}$ assessment (i.e. $\widetilde{N_{z h}}$ ) according to annual heating-period meteorological condition and actual heating region situation (as shown in Figure 1-A);

Step 2: Compute heat demand by integrating the heat load curve over the fuzzy heat duration endpoint inputs at $\alpha$-cut $=1$ and 0 (as shown in Figure 1-B);

Step 3: Formulate the fuzzy heat demand with obtained heat demands at $\alpha$-cut $=1$ and 0 , which possess the same fuzzy membership degree shape as the fuzzy total heating duration (as shown in Figure 1-C).

For predicting the heat demand parts undertaken by the different heat sources in the MHSCHS, a supplementary calculation procedure needs to be added into Step 2, which is to use the given thermalization coefficient (TC) to compute the corresponding heat demand parts with the fuzzy heat duration.

\section{CASE STUDY}

The study area named as Shenbei new district is located in the north of metropolis of Shenyang, of which the heating period is approximately 5 months. The corresponding spaceheating area covered by the MHSCHS is identified as $5.97 \times 10^{6}$ $\mathrm{m}^{2}$, according to Shenyang census data. The MHSCHS is basically consist of a coal-fired combined heat and power (CHP) as the main heat source (MHS) and several peakshaving boilers as the heat supply sources for peak compensation (PHS). In this region, the ratio of ordinary heatsupply area to the energy-conservation area is 6.5: 3.5 and their corresponding heating load indices are respectively 64 and 45 $\mathrm{W} / \mathrm{m}^{2}$. The outdoor average temperatures during the annual heating period is shown in Figure 2, and the total outdoor average temperature, design outdoor and indoor temperature is respectively $-5.7^{\circ} \mathrm{C},-19^{\circ} \mathrm{C}$ and $18^{\circ} \mathrm{C}$. With the purpose of energy saving and environment protection, the heating duration should be evaluated depending on the annual practical meteorological condition, and can be expressed as trapezoidal fuzzy number, which is $\widetilde{N_{z h}}=(140,165,150,155)$ on the basis of investigation from previous MHSCHS managers and relative experts. Through the aforementioned method in sector 2 , the fuzzy heating demand parts undertaken by different heat 
sources can be subsequently identified under different

$\alpha$-cut levels). combinations of various TCs and system possibility levels (i.e.

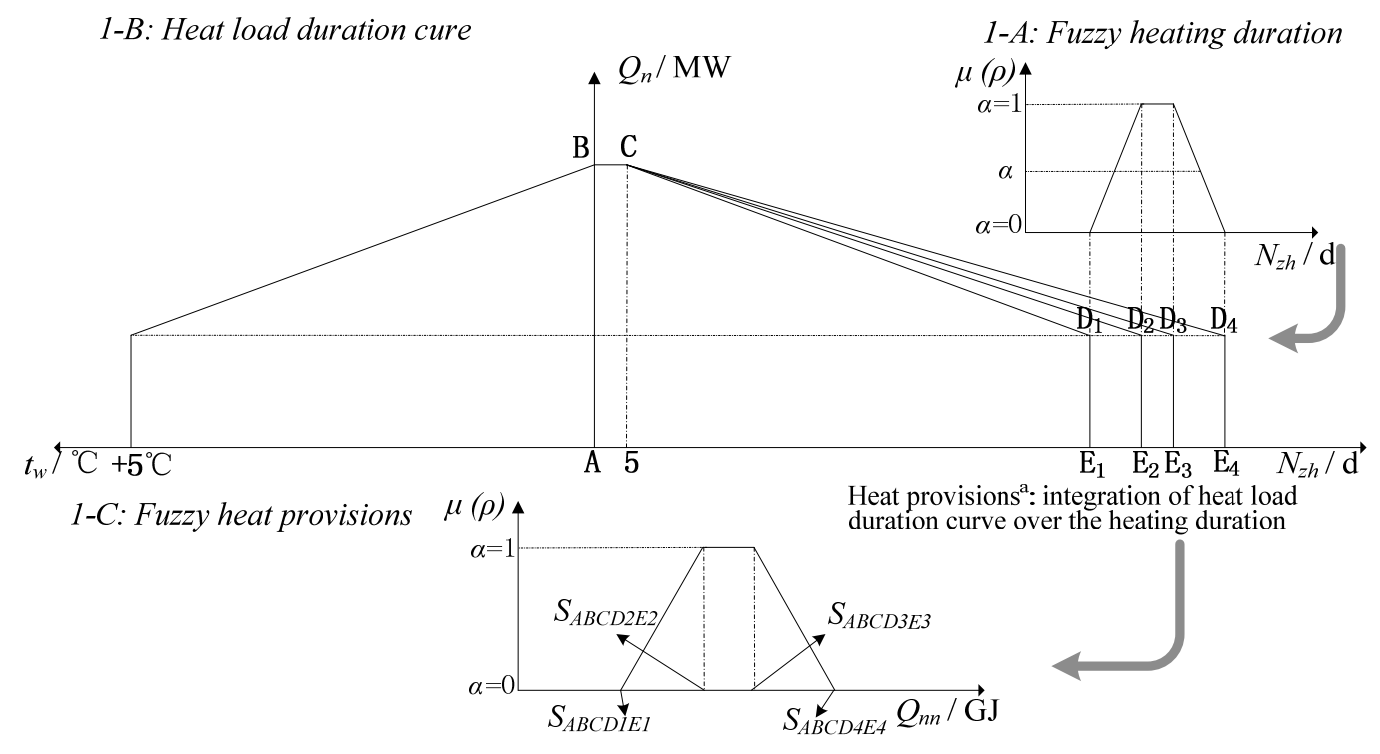

FIGURE I. SCHEMATIC OF FUZZY HEAT DEMAND FORMULATION PROCESS.

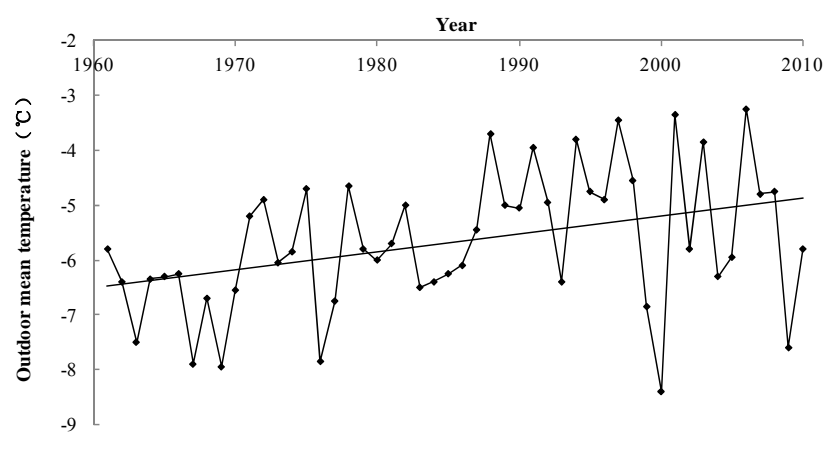

FIGURE II. ANNUAL OUTDOOR AVERAGE TEMPERATURES DURING HEATING PERIOD.

\section{RESUlt ANALYSIS}

In this investigation, the corresponding TC values (i.e. $\beta$ ) are $0.45,0.5,0.55,0.6$ and 0.65 because the relative national regulations and policies require the $\mathrm{TC}$ of the residential MHSCHS system to be in the range from 0.5 to 0.6 , and occasionally beyond this range if emergencies occur. Meanwhile, according to Zimmermann's fuzzy set theory, different membership degrees represent the predetermined different system state possibility levels, and a group of heatdemand interval values can be obtained for different heat sources based on the $\alpha$-cut quantitation technique [9]. Therefore, in order to quantitatively identify the influence of fuzzy uncertainty on the heat demands, the $\alpha$-cut levels are proposed to be equal to $0.2,0.5$ and 0.8 , corresponding to relatively-low, moderate and relatively-high system possibility levels, respectively. With the different the various parameter combination inputs (i.e. different $\beta$ and $\alpha$ ), the results of the space heat demand parts undertaken by different heat sources are illustrated in Figure 3 based on the method developed in Section 2.

Since the developed heat demand prediction model is based on the integration of the fuzzy set theory and HLDC method, the uncertainty information can be directly reflected in the prediction results. In Figure 3, the abbreviation of UB and LB represent the upper and lower bounds of the heat demand prediction results and it can be found that all the results are expressed as interval numbers. For instance, under the combination of $\alpha=0.5$ and $\beta=0.55$, the heat demand parts undertaken by MHS and PHS are respectively [2.20, 2.43] and $[0.61,0.66]$ PJ. Therefore, compared with conventional deterministic method (i.e. HLDC method), the advanced method cannot only deal with the fuzzy uncertainty associated with the heating system parameters quantitatively, but can also provide potential fluctuant range of the heat demand under different system possibility levels, from which a mass of prediction alternatives can be achieved for the MHSCHS manager to refer to.

From the point of system possibility view, it can be seen that with the rising $\alpha$-cut values, the UBs and LBs of the heat demand parts undertaken by both MHS and PHSs present inverse variation trends. Specifically, the UBs decrease while the LBs increase. For example, when $\beta$ is at 0.55 , the UBs of the heat demands undertaken by the MHS would be 2.48, 2.43 and 2.38 PJ, corresponding to $\alpha=0.2,0.5$ and 0.8 , respectively. In comparison, the obtained LBs would be 2.16, 2.20 and 2.25 PJ. This implies that the heat demand interval (i.e. the difference between the UB and LB) originated from the $\alpha$-cut quantification means is gradually narrowed when the system possibility level is enhanced. On the other hand, from the 
MHSCHS operation perspective, as shown in Figure 3, it can be seen that the UBs and LBs of the heat demand parts undertaken by the MHS would both augment while the heat demand parts undertaken by the PHSs would decline during the TC variation process from 0.45 to 0.65 . For instance, at $\alpha=0.5$, the heat demand intervals of the MHS are [1.88, 2.08], [2.05, $2.26],[2.20,2.432],[2.34,2.58]$ and $[2.45,2.70] \mathrm{PJ}$ under $\beta=$ $0.45,0.5,0.55,0.6$ and 0.65 , respectively. On the contrary, the heat demand intervals of the MHS would decline from [0.92, $1.01]$ to $[0.36,0.39] \mathrm{PJ}$ when $\beta$ varies from 0.45 to 0.65 . It is thus indicated that the changing trends of the heat demand parts undertaken by the MHS and the PHSs are in opposite direction. That is mainly because when $\beta$ increases, the higher heat load is assigned to the MHS, and leads to more heat demand parts undertaken by the MHS.

In general, the developed prediction method can directly generate the heat provision alternatives from prediction intervals for different heat sources at different system operation modes, and moreover, it has the capability of providing the tradeoff analysis between the system possibility and the interval size of the prediction results, which is far beyond the capability of the traditional HLDC method.

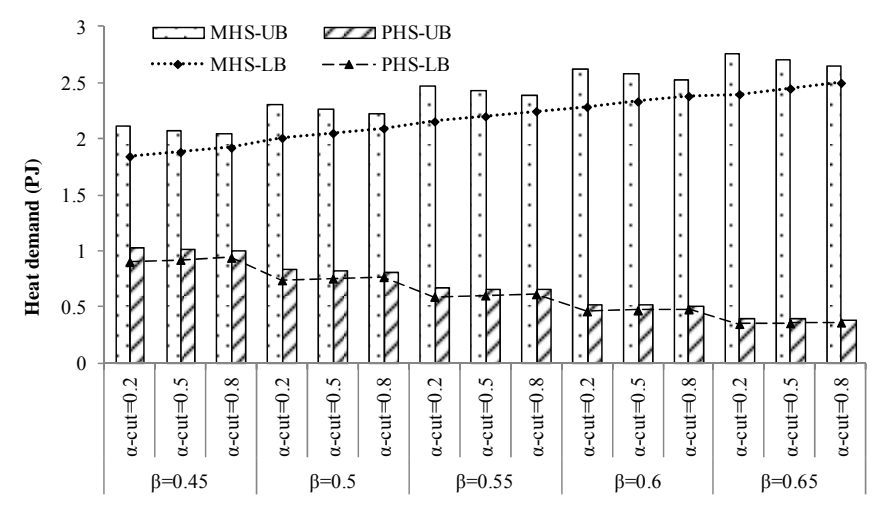

FIGURE III. HEAT DEMAND PREDICTION RESULTS UNDER DIFFERENT COMBINATIONS OF THERMALIZATION COEFFICIENTS AND SYSTEM POSSIBILITY LEVELS.

\section{CONCLUSION}

Compared with the conventional prediction methods, this work provides a residential space-heating demand prediction method which can effectively cope with the fuzzy uncertainty. Through $\alpha$-cut-based quantification means, the developed method can generate the space-heating demand interval results at various system possibility levels. It is indicated that the higher system possibility is, the narrower the prediction interval becomes, in which the decision maker can adjust the practical heat provisions of the heat sources. Moreover, with the incremental thermalization coefficient, the opposite variation trend for the MHS and PHS heat provisions can be obtained, which is that the heat provision for the MHS would increase while the PHS heat provision would decrease.

Due to the integration of the fuzzy set theory and HLDC method into the developed method, it is helpful to provide the relative MHSCHS managers a potential tradeoff analysis between the system possibility and the selectable heat provision ranges of different heat sources. Besides, the developed method can also directly generate heat provisions for different heat sources based on the adjustable TC input. Therefore, the obtained results can be served as the heat provision reference or the boundary condition inputs to the subsequent MHSCHS energy management and optimization for the further analysis under uncertainty.

\section{ACKNOWLEDGMENT}

This research was supported by the Program for Onehundred Talent Program of the Chinese Academy of Sciences (Y5AA100A01)

\section{REFERENCES}

[1] D.Z. Fu; G.H. Huang, "Factorial-interaction-based economic optimization research for combined heat and power plant," J. Nor. Chi. Elec. Pow. Uni., vol. 41(4), pp. 101-106, July, 2014.

[2] P. Milan, S. Shahaboddin, H.A. Mohammad, et al., "Appraisal of soft computing methods for short term consumers' heat load prediction in district heating systems," Energy, vol. 82(15), pp. 697-704, March, 2015

[3] S. Shahaboddin, P. Dalibor, E. Rasul, et al., "Heat load prediction in district heating systems with adaptive neuro-fuzzy method," Renew. Sust. Energ. Rev., vol. 48, pp. 760-767, August, 2015.

[4] C. Tiberiu, I. Vlad, C. Bogdan, "Multiple regression model for fast prediction of the heating energy demand," Energ. Buildings, vol. 57, pp. 302-312, February, 2013

[5] I. Nima, C.O. Hwai, S. Shahaboddin, G. Hossein, W.T. Chong, "Intelligent forecasting of residential heating demand for the District Heating System based on the monthly overall natural gas consumption," Energ. Buildings, vol. 104(1), pp. 208-214, October, 2015.

[6] M. Daša, I. Laure, V. Henk, "Statistical model of the heating prediction gap in Dutch dwellings: Relative importance of building, household and behavioural characteristics," Energ. Buildings, vol, 105(15), pp. 43-59, October, 2015.

[7] H.C. Wang, W.L Jiao, R. Lahdelma, P.H. Zou, "Techno-economic analysis of a coal-fired CHP based combined heating system with gasfired boilers for peak load compensation," Energ. Policy, vol. 39(12), pp. 7950-7962, December, 2011.

[8] D.Z. Fu, G.H. Huang and Y.M. Liu, "Optimization Study of Coal Blending Based on Heat supply and Power Generation Analysis," Elec. Pow. Constru., Vol.34, pp. 71-76, November, 2013.

[9] H.J. Zimmermann, Fuzzy set theory and its applications, 3rd ed., Norwell, MA, USA, 1996. 